Research, Society and Development, v. 9, n. 11, e53891110277, 2020

(CC BY 4.0) | ISSN 2525-3409 | DOI: http://dx.doi.org/10.33448/rsd-v9i11.10277

\title{
Abortamento de repetição: etiologia e cuidados
}

Recurrent abortion: etiology and care

Aborto repetido: etiología y cuidados

Recebido: 17/11/2020 | Revisado: 17/11/2020 | Aceito: 20/11/2020 | Publicado: 25/11/2020

\section{Breno Pena Barros}

ORCID: https://orcid.org/0000-0003-3035-7193

Centro Universitário FAMETRO, Brasil

E-mail: brenobarros_7@hotmail.com

Carolynne Hernandez Barbosa

ORCID: https://orcid.org/0000-0003-1833-5194

Centro Universitário FAMETRO, Brasil

E-mail: caroljudogremio@outlook.com

Ellen Anny Pontes Moura dos Santos

ORCID: https://orcid.org/0000-0001-7653-6985

Centro Universitário FAMETRO, Brasil

E-mail: ellenannypontes@hotmail.com

Esmeson Pereira da Costa

ORCID: https://orcid.org/0000-0003-2844-6496

Centro Universitário FAMETRO, Brasil

E-mail: esmeson.ep@gmail.com

Graciana de Sousa Lopes

ORCID: https://orcid.org/0000-0003-3615-9040

Centro Universitário FAMETRO, Brasil

E-mail: gracilopess@hotmail.com

Roseane Gomes Ribeiro

ORCID: https://orcid.org/0000-0002-2426-9106

Centro Universitário FAMETRO, Brasil

E-mail: rosegomes452@gmail.com

Welberson Soares de Souza

ORCID: https://orcid.org/0000-0001-5307-268X

Centro Universitário FAMETRO, Brasil

E-mail: welberson2694@gmail.com 


\title{
Resumo
}

Objetivo: discutir os principais aspectos relacionados ao abortamento de repetição e cuidados preventivos e caracterizar a importância da assistência do pré-natal na prevenção ao abortamento de repetição. Método: Trata-se de um estudo qualitativo de caráter explicativo através de revisão integrativa de literatura, que é um método que proporciona conhecimento e resultados de estudos na prática, com ampla abordagem metodológica incorporando conceitos, revisão de teorias, evidencias e analise de problemas metodológicos. Resultados: o aborto recorrente pode ser de diferentes etiologias, porém, 40 a 60\% dos abortos recorrentes são de causas identificáveis ou idiopáticos pelo fato de não estarem ligados a nenhuma doença ou talvez por falta de investigação. Conclusão: as perdas gestacionais recorrentes são multifatoriais, podendo ocorrer por anormalidades nos cromossomos e útero, infecções e causas que podem ser consideradas como identificáveis por não terem ligação a uma doença especifica. Porém, a assistência pré-natal é de grande importância na prevenção ao aborto espontâneo de repetição, pois a mesma planeja a redução de riscos e garante o bem estar da mulher em seu período gravídico.

Palavras-chave: Saúde da Mulher; Câncer de Colo Uterino; Exame citopatológico.

\begin{abstract}
Objective: this article aims to discuss the main aspects related to recurrent abortion and preventive care and to characterize the importance of prenatal care in preventing recurrent abortion. Method: This is a qualitative study of an explanatory character through an integrative literature review, which is a method that provides knowledge and results of studies in practice, with a broad methodological approach incorporating concepts, review of theories, evidence and analysis of methodological problems. Results: recurrent abortion can be of different etiologies, however, 40 to $60 \%$ of recurrent abortions are of identifiable or idiopathic causes due to the fact that they are not linked to any disease or perhaps due to lack of investigation. Conclusion: recurrent pregnancy losses are multifactorial, and can occur due to abnormalities in the chromosomes and uterus, infections and causes that can be considered as identifiable because they are not linked to a specific disease. However, prenatal care is of great importance in preventing recurrent spontaneous abortion, as it plans to reduce risks and guarantee the well-being of women during their pregnancy.
\end{abstract}

Keywords: Women's Health; Cervical Cancer; Cytopathological Examination. 


\section{Resumen}

Objetivo: este artículo tiene como objetivo discutir los principales aspectos relacionados con el aborto recurrente y la atención preventiva y caracterizar la importancia de la atención prenatal en la prevención del aborto recurrente. Método: Se trata de un estudio cualitativo de carácter explicativo a través de una revisión integradora de la literatura, que es un método que aporta conocimientos y resultados de estudios en la práctica, con un enfoque metodológico amplio incorporando conceptos, revisión de teorías, evidencia y análisis de problemas metodológicos. Resultados: el aborto recurrente puede ser de diferentes etiologías, sin embargo, del 40 al 60\% de los abortos recurrentes son de causas identificables o idiopáticas por no estar vinculados a ninguna enfermedad o quizás por falta de investigación. Conclusión: las pérdidas de embarazos recurrentes son multifactoriales y pueden ocurrir por anomalías en los cromosomas y útero, infecciones y causas que pueden considerarse identificables por no estar vinculadas a una enfermedad específica. Sin embargo, la atención prenatal es de gran importancia para prevenir el aborto espontáneo recurrente, ya que planea reducir los riesgos y garantizar el bienestar de la mujer durante su embarazo.

Palabras clave: Salud de la mujer; Cáncer de cuello uterino; Examen citopatológico.

\section{Introdução}

De acordo com a Organização Mundial da Saúde (OMS), o abortamento é a interrupção da gravidez que pode acontecer antes da $22^{\mathrm{a}}$ semana ou que tenha o peso fetal menor que $500 \mathrm{~g}$. $\mathrm{O}$ aborto pode ser dividido em precoce, que ocorre ate $12^{\mathrm{a}}$ semanas e o tardio que acontece entre a $13^{\mathrm{a}}$ e a $22^{\mathrm{a}}$ semana de gestação, ou seja, no primeiro trimestre (Ribeiro \& Alencar Júnior, 2013). O aborto espontâneo precoce é considerado uma situação comum, e em muitos casos, o aborto acontece antes mesmo de a gravidez ser descoberta.

Abortamento de repetição (AR), perda recorrente da gravidez (RLP) e aborto espontâneo recorrente (RSA), são termos utilizados para se referir ao processo de abortamento que tenha ocorrido três ou mais vezes pela mesma mulher (NO, 2011). Uma pesquisa recente sobre o assunto relata as características epidemiológicas atestando um aumento significativo na incidência nos últimos 10 anos e relacionando a exposição ambiental e comportamental, e o aumento da idade materna na concepção (Rasmark et al., 2017).

Os profissionais que estão na área materna e obstétrica tem o prazer de recepcionar a vida e presenciar a alegria da chegada de um novo ser, porém existem situações de infortúnio, e uma dessas ocorrências é o aborto espontâneo que ali interrompe de forma inesperada e 
triste uma gravidez, que pode ser o sonho daquela mulher. O aborto por repetição pode ser um processo ainda mais doloroso para aquela mulher, ou casal que já tentou a gravidez por várias vezes, porém sem êxito. Os profissionais da área de saúde precisam conhecer melhor o processo de abortamento e as suas várias das causas, assim também seu diagnóstico para assim poder prestar os devidos cuidados e atenção.

O abortamento espontâneo recorrente tem sido bastante comum e por isso, essa pesquisa procurou juntar informações com a finalidade de responder o seguinte problema de pesquisa: quais são as principais causas do abortamento de repetição? Quais os motivos que essas mulheres não conseguem levar a gravidez até o final?

O objetivo geral do presente trabalho é discutir os principais aspectos relacionados ao abortamento de repetição e cuidados preventivos e caracterizar a importância da assistência do pré-natal na prevenção ao abortamento de repetição.

\section{Referencial Teórico}

\section{Processo de abortamento}

Segundo o Manual Técnico de Gestação de Alto Risco do Ministério da saúde, o aborto é o produto eliminado no processo de abortamento. Já o abortamento é definido como a interrupção da gravidez que pode acontecer antes da $22^{\mathrm{a}}$ semana ou que tenha o peso fetal menor que $500 \mathrm{~g}$. No cenário mundial a perda gestacional varia entre 15 a $20 \%$ das gestações confirmadas, o processo de abortamento é algo comum entre as mulheres brasileiras ocorrendo a maior parte antes da $12^{\mathrm{a}}$ semana de gestação (Camayo \& Martins \& Cavalli, 2011).

O Abortamento Espontâneo de Repetição (AER) é definido como uma síndrome que é caracterizada por três ou mais episódios de aborto consecutivos e espontâneos (Stirrat, 1990). Porém, a Sociedade Americana de Medicina Reprodutiva, recentemente redefiniu-a como duas ou mais perdas gestacionais fundamentando-se na predominância das prováveis causas (Asrm, 2013).

\section{Processo de abortamento}

O aborto pode ser classificado como espontâneo, quando o processo de abortamento principia independentemente de qualquer procedimento ou prática externa, normalmente 
acontece devido a problemas de saúde da gestante ou mesmo do feto. A outra classificação é o aborto induzido ou provocado, ocorre quando é utilizado qualquer tipo de método abortivo externo, que pode ser químico ou mecânico, pode ocorrer incitamento voluntário ou involuntário da gestante, é um procedimento considerado ilegal. Existe também, o aborto que é considerado legal (Lei $n^{\circ}$ 2.848), quando não houver alternativa para salvar a vida da gestante ou quando a mulher foi vítima de estupro e que resultou na gravidez, nesses casos com o consentimento da mulher ou do responsável (no caso de menor), não é crime. Em uma pesquisa realizada com 100 mulheres, constou-se que $11 \%$ delas relataram ter realizado aborto provocado, e 89\% mencionaram ter sofrido aborto espontâneo (Borsari et al, 2013).

\section{Fatores Preditores}

Pesquisas apontam que 2 a 4\% dos casais são afetados pelo Abortamento Espontâneo de Repetição (AER), porém apenas metade consegue encontrar uma causa específica, a literatura aponta como uma das dificuldades os métodos diagnósticos que ainda são ineficientes. Entre as causas que são mais comuns de ser encontradas, estão as alterações cromossômicas, que é o causador de 40-80\% dessas perdas (Morales, 2008).

Bender (2018) e outros autores, citam algumas outras causas que também podem estar relacionadas, que são as anomalias uterinas, os distúrbios endócrinos, resposta imune, e outras ainda não comprovadas que são as infecções e toxinas ambientais (como tabagismo e consumo excessivo de álcool); assim como, idade da gestante avançada e o excesso de peso.

1. Anormalidades cromossômicas: é responsável por 2 a $4 \%$ das perdas recorrentes, faz se necessário a avaliação do cariótipo padrão do casal, apesar de sua baixa porcentagem

2. Anormalidades uterinas: podem ser adquiridas ou congênitas. Adquiridas: sinéquias intrauterinas (cicatriz que se dá no revestimento interno do útero); leiomiomas submucosos e intracavitários (são nódulos benignos que se localizam na parte interna do útero); pólipos endometriais (geralmente são benignos e fixam-se na parede intrauterina). Congênitas: malformações Mullerianas (ocorrem por fusão embriológica defeituosa), como útero bicorno (tem forma de coração), unicorno (o tecido uterino não se forma direito e o órgão tem a metade do tamanho e apenas uma tuba uterina) (Jaslow \& Kutteh, 2013).

3. Síndrome do anticorpo antifosfolípide (SAF): é um fator imunológico autoimune que pode causar coágulos sanguíneos. O SAF é único distúrbio autoimune que está ligado à perda gestacional (geralmente com 10 semanas e está associados à morte fetal, parto prematuro, pré-eclâmpsia e restrição de crescimento fetal) (Ye, 2017). 
Research, Society and Development, v. 9, n. 11, e53891110277, 2020

(CC BY 4.0) | ISSN 2525-3409 | DOI: http://dx.doi.org/10.33448/rsd-v9i11.10277

Outras causas que também podem ser associadas são: fatores infecciosos (dentre as causas de perda gravídica recorrente a etiologia infecciosa está ligada 2,4\% dos casos. Em um estudo realizado, a endometrite crônica foi uma evidencia encontrada em mulheres que tiveram perdas gestacionais recorrentes, o que pode indicar que esse processo infeccioso pode se desenvolver no interior do útero o que é desfavorável ao feto); Fator Imunológico (também conhecido como aloimunes, que são as reações imunológicas, estudos sugerem um desequilíbrio entre a imunidade celular Th1/Th2 nas mulheres com aborto recorrente); trombofilia hereditária (alteração na coagulação sanguínea podendo causar trombose te pode ser referente ao AR ao desencadear uma resposta hemostática materna inadequada); e por último as causas masculinas (que estar interligado ao DNA espermático, aneuploidias e alterações na cromatina dos espermatozoides) (Rauber et al, 2018).

\section{Condutas relacionadas ao processo de abortamento}

Sabemos que o AR pode ocorrer por várias causas, e por esse motivo existe mais de um tipo de tratamento. O procedimento para esvaziamento uterino inclui desde a conduta ativa até a expectante. A ativa consiste naquela em que se utiliza os fármacos como, por exemplo, o misoprostol que pode ser oral, sublingual ou vaginal associado ou não ao mifepristonel, o que depende da idade gestacional; métodos cirúrgicos, como aspiração manual intrauterina (AMIU), que pode ser a vácuo ou elétrico para gestação de até 12 a 14 semanas; e por último a curetagem que é mais utilizada em gravidez mais avançada (Organização Mundial da Saúde, 2013). A conduta expectante resulta na completa expulsão e pode ter intermitência até a expulsão completa, esse é um procedimento com risco de infecção e hemorragia.

A curetagem uterina é um procedimento cirúrgico onde ocorre o esvaziamento da cavidade endometrial por meio de uma cureta ou dispositivo de aspiração. É um método que pode haver algumas complicações, como: perfuração uterina, lesão do colo do útero, infecção, hemorragia, aderência intrauterina e o esvaziamento incompleto. Também existem riscos que podem ser evitados, esses ricos são: distúrbios de coagulação gestação implantada fora do útero (ectópica), infecção pélvica (Febrasgo, 2010). 


\section{Metodologia}

Trata-se de um estudo qualitativo de caráter explicativo através de revisão integrativa de literatura, com a finalidade de conhecer as no período de 2010 a 2020. Foram estabelecidas as bases de dados Scientific Eletronic Library on Line (SciELO), Biblioteca Virtual em Saúde - BVS e Literatura Latino-Americana e do Caribe em Ciências da Saúde (LILACS). Para a busca na base de dados utilizando os seguintes descritores: aborto espontâneo recorrente, interrupção espontânea da gravidez, fatores relacionados. Os artigos foram identificados conforme as principais causas do aborto recorrente, visando às estratégias de redução para essa prevalência. Foram adotados os seguintes critérios de elegibilidade artigos científicos completos em português, disponíveis gratuitamente nas plataformas de busca de dados dos anos de 2010 a 2020 e como critério de inelegibilidade artigos em formato de resumos, artigos em espanhol e inglês, artigos que abordam a temática, teses de Doutorado e dissertações de Mestrado.

Os artigos foram submetidos a uma leitura rigorosa do texto completo, incluindo-os ou excluindo-os, de acordo com os critérios de seleção para identificar as principais causas do abortamento de repetição, analisando os artigos científicos de acordo com os seguintes aspectos: Titulo Autores, Ano/País/Base de Dados, Delineamento do estudo/ Nível de Evidência/ Instrumento, Síntese da conclusão e Resultados.

\section{Resultados e Discussão}

$\mathrm{Na}$ amostra final desta pesquisa foram utilizados 14 artigos científicos. Esse estudo tem como objetivo discutir as principais causas relacionadas ao abortamento de repetição e a importância da assistência pré natal na prevenção de AR. Dos artigos em estudo um foi publicado no ano de 2020 e um em 2019, dois em 2018, e dois em 2017, em 2015 um e no ano de 2013 foram publicados três, nos anos 2011 e 2010 foram publicados dois artigos em cada ano. 
Figura 1: Fluxograma: Coleta de dados.

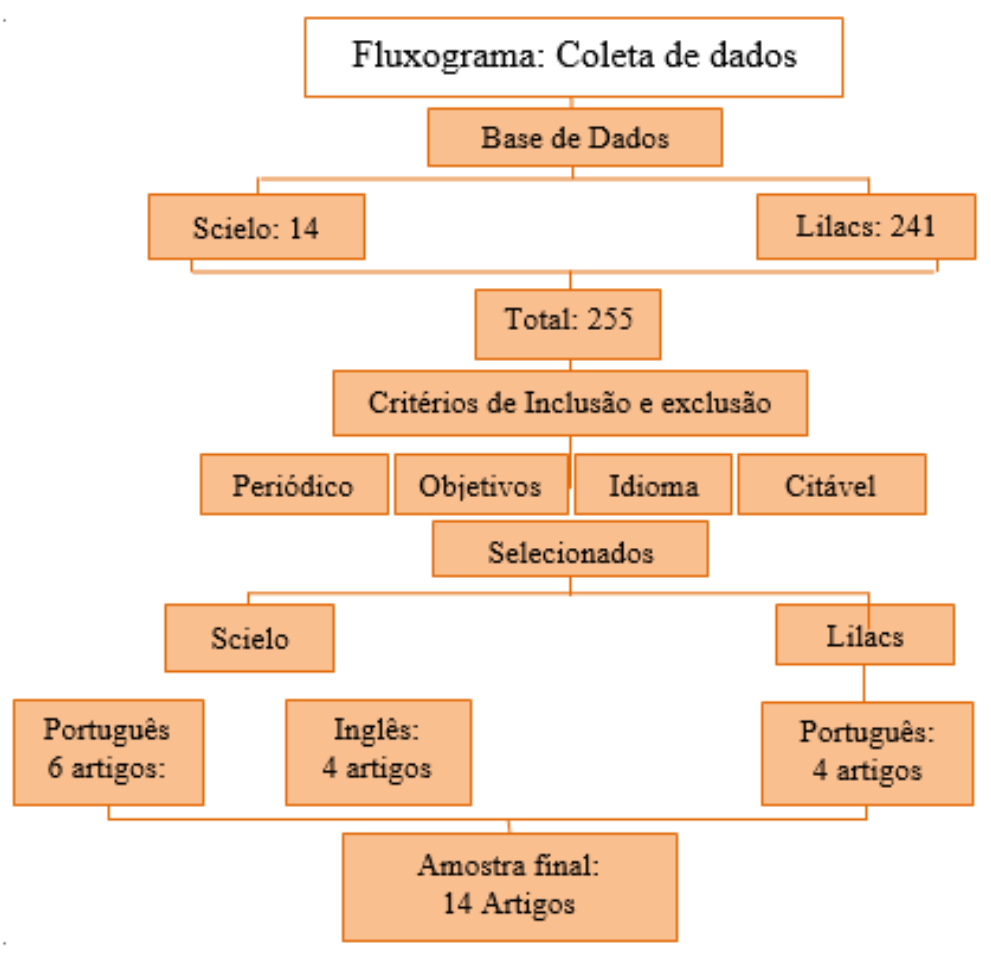

Fonte: Elaborado pelos acadêmicos, (2020).

\section{Aspectos relacionados ao aborto de repetição}

De acordo com Machin et al (2019), Ramos et al (2010), Li et al (2017) e Souza (2011) aborto é a intercepção ou o fim de uma gravidez, quando ocorre até a $22^{a}$ semana de gestação, que tenha o peso menor que $500 \mathrm{~g}$ ou que seja menor que $16 \mathrm{~cm}$. Os autores classificam o aborto como induzido e espontâneo. O aborto espontâneo é quando não há associação com prática externa, podendo estar relacionada a problemas saúde materno ou fetal e induzido quando a grávida com seu consentimento ou não utiliza algum método para expulsar o feto, podendo ser químico através de drogas ou mecânico. Ramos (2010), em sua pesquisa, entrevistou algumas mulheres que passaram pelo processo de abortamento e constatou que das mulheres em estudo que 55,7\% delas haviam passado pelo aborto com apenas 12 semanas de gravidez ou menos e que 56,3\% eram de causa identificável e o restante foram induzidos.

É considerado como aborto de repetição, segundo Li et al (2017), Marqui (2018), Rauber et al (2018), Junior et al (2010) e Oliveira (2011), quando esse episódio acontece por três ou mais vezes consecutivas, podendo ser por múltiplos fatores.

Porém, o autor Souza et al (2011) em sua pesquisa fala que pode ser considerado 
aborto recorrente a partir da segunda perda gestacional consecutiva. $\mathrm{O}$ mesmo justifica que diminuindo assim o número de perdas gestacionais, possibilita a investigação precoce e consequentemente o tratamento precoce, permitindo a mulher ter a chance de ter uma gravidez com sucesso.

Como já foi colocado anteriormente, o AR pode ser de diferentes etiologias, Marqui (2018) em seu artigo diz que 40 a 60\% dos abortos recorrentes são de causas identificáveis ou idiopáticos pelo fato de não estarem ligados a nenhuma doença ou talvez por falta de investigação. Entretanto, dentre os abortos recorrentes com causas associáveis estão às anomalias cromossômicas, que segundo ele podem ser numéricas (alteração de números dos cromossomos) ou estruturais (modificações morfológicas).

O autor Rauber et al., (2018), acrescenta que dentre as principais causas, estão as anomalias cromossômicas parentais, como por exemplo a SAF e algumas anormalidades uterinas. Souza et al., (2011), concorda e diz que em sua pesquisa utilizando o exame de histerscopia foi possível observar que $33 \%$ das mulheres em pesquisa tinham algum tipo de anomalias uterinas, tanto congênitas como adquiridas. Li et al., (2017) por sua vez, destaca as etiologias autoimunes como uma das principais em abortos espontâneos de repetição.

Conforme Junior et al., (2010) cita as causas infecciosas, porém apenas 2,4\% das mulheres em processo de abortamento tem sua perda gestacional relacionada as infecções. A Chlamydia trachomatis (CT) é uma bactéria e um dos agentes infecciosos mais examinados, no entanto devido sua desaparição no momento da perda gestacional, torna sua associação a perda gestacional ainda mais difícil.

\section{Aspectos relacionados ao aborto de repetição}

Feliciano et al., (2013), em seu estudo enfatiza a importância das consultas de pré natal pois objetiva a redução de riscos e a promoção a saúde da mãe e do feto. Sehnem et al., (2020) e Moura et al., (2015), por sua vez falam que para a redução de ricos as consultas precisam ser iniciada o mais precoce possível, segundo a literatura o ideal que inicie até 120 dias, pois assim é possível identificar e tratar alguma patologia que venha ocorrer durante o período gravídico, evitando assim o aborto espontâneo. Durante as consultas assistenciais é realizada a solicitação e avaliação de exames laboratoriais que deve ser feito duas vezes (no início do pré natal, no início do terceiro trimestre ou quando necessário), testes rápidos para sorologia, ultrassonografia para avaliação do estado situacional do bebê, aferição dos SSVV, medição da altura uterina, ausculta dos batimentos cárdico fetal, suplementação e também as 
Research, Society and Development, v. 9, n. 11, e53891110277, 2020

(CC BY 4.0) | ISSN 2525-3409 | DOI: http://dx.doi.org/10.33448/rsd-v9i11.10277

orientações quanto aos cuidados que a gestante deve ter, quanto a alimentação, atividades físicas, uso de cigarros e bebidas alcoólicas, descanso e também a auto medicação.

Figura 2: Fluxograma de Pré Natal.

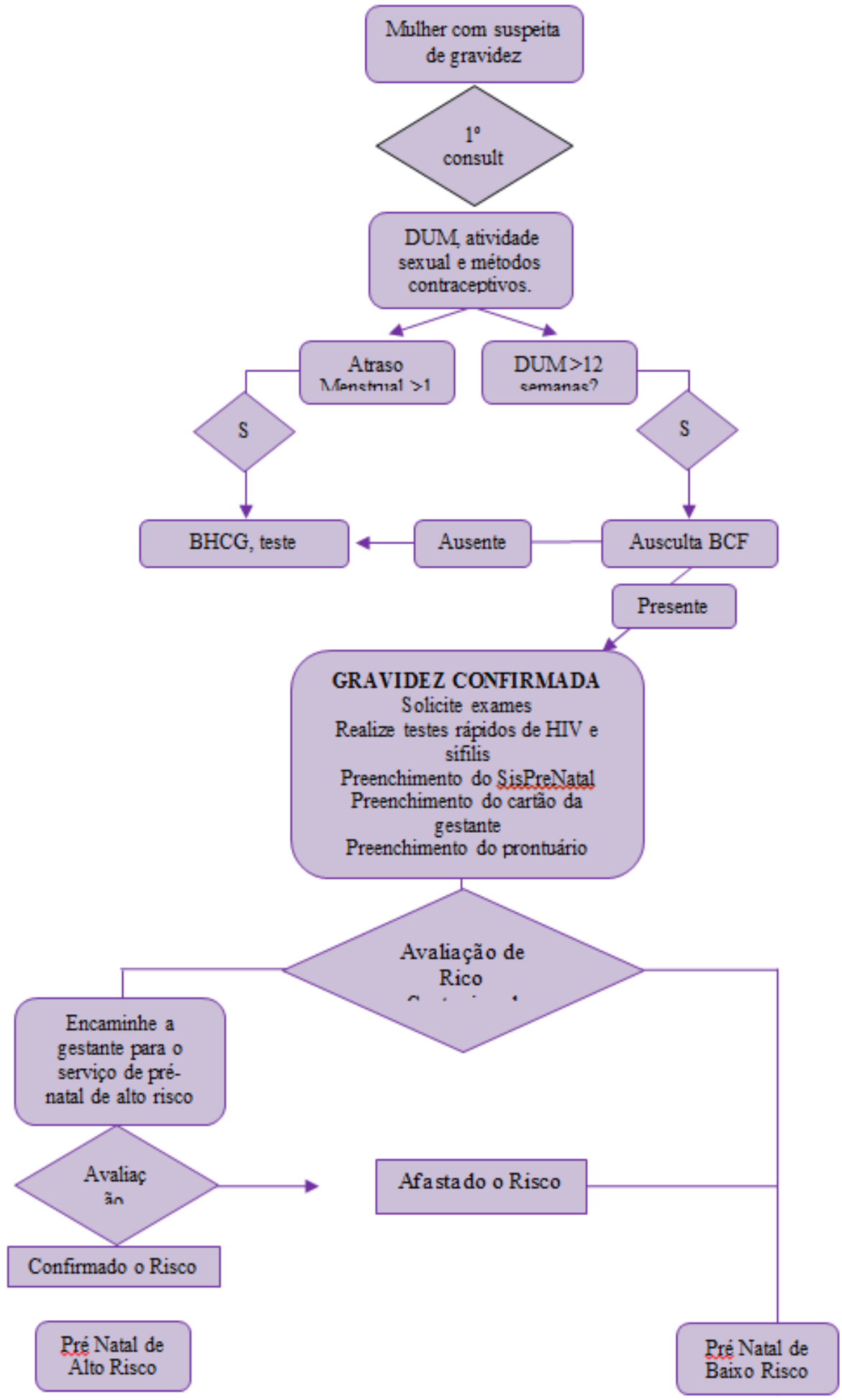

Fonte: Elaborado pelos acadêmicos, (2020). 
Nesse sentido, Peixoto (2014), fala no Manual de assistência ao pré natal o objetivo central da assistência no período gravídico, que é o bem estar da gestante, tanto físico como psíquico durante todo o processo gestacional, minimizando riscos para a mãe e para o bebê. Dessa forma, a anamnese e o exame físico são de extrema importância, pois permitem reconhecer de imediato as gestantes com alguma doença, seu estado atual e os riscos inerentes ao quadro, podendo assim fazer um tratamento precoce e evitar possíveis perdas.

Para Brasil (2012), fala sobre os 10 passos para um pré natal de qualidade na atenção Básica. O $1^{\circ}$ deles é a Captação precoce da gestante, devendo iniciar as consultas até $12^{\mathrm{a}}$ semana de gestação; $2^{\circ}$ passo é oferecer a cliente recursos, sejam eles, físicos, materiais e técnicos; $3^{\circ}$ passo a gestante deve ter garantido a solicitação e realização de exames, assim como o resultado e a sua avaliação; $4^{\circ}$ passo deve-se promover a gestante e a seu acompanhante, o acolhimento humanizado, escutando-os, sejam questões emocionais, intelectuais ou socioculturais (roda de gestantes); $5^{\circ}$ passo, quando for preciso, a gestante tem o direito de transporte público gratuito para o atendimento de pré natal; no $6^{\circ}$ passo o ministério da saúde fala que o parceiro tem direito a consultas, exames e acesso a informações durante e após a gravidez; $7^{\circ}$ passo, deve ter alcance a unidade especializada, se assim for preciso; $8^{\circ}$ passo, incentivar o parto natural; $9^{\circ}$ passo a gestante tem o direito de conhecer a unidade em que vai ocorrer o parto; $10^{\circ}$ e último passo as mulheres devem conhecer e exercer os direitos garantidos por lei.

Moura et al., (2015), por sua vez fala da qualidade da assistência e a qualidade do profissional enfermeiro, pois o mesmo é um dos principais diante as consultas de pré natal. Segundo o Decreto $n^{\circ}$ 94.406/87, que regulamenta a Lei $n^{\circ} 7.498$, de 25 de junho de 1986, que permite o profissional enfermeiro a realizar consultas de pré natal e sua prescrição assistencial e medicamentosa conforme estabelecido nos programas de saúde assim como prestação de assistência de enfermagem à gestante, parturiente, puérpera e ao recém-nascido (Brasil, 1987).

Gomes et al., (2019), em sua pesquisa fala sobre a percepção das gestantes quanto as consultas de enfermagem no pré natal e o acolhimento é um dos itens principais para elas, pois é a partir daí que elas criam vínculos e passam a confiar mais no profissional. Um sorriso, a escuta, a atenção, são itens básicos, porém, de extrema importância para as gestantes e seus acompanhantes, sendo também um incentivo a retornarem as consultas. 


\section{Considerações Finais}

Ao chegarmos ao fim deste estudo, consideramos que as perdas gestacionais recorrentes são multifatoriais, podendo ocorrer por anormalidades nos cromossomos e útero, infecções e causas que podem ser consideradas como identificáveis por não terem ligação a uma doença especifica. Porém, a assistência pré-natal é de grande importância na prevenção ao aborto espontâneo de repetição, pois a mesma planeja a redução de riscos e garante o bem estar da mulher em seu período gravídico. Durante a as consultas assistenciais é possível fazer a detecção de riscos e doenças e assim iniciar o tratamento precoce e evitar possíveis problemas graves e perdas.

Todos os objetivos propostos neste estudo foram alcançados, possibilitando discutir as principais causas do abortamento de repetição e relatar a importância da assistência pré natal a essas mulheres que já sofrem com o aborto, porém continuam com o desejo de gerar e desta forma poder presta uma assistência de qualidade e humanizada para prevenção e a promoção da saúde da mamãe e do feto. Para tanto, foi coletado dados de diversas fontes para assim atingir os objetivos estabelecidos.

O aborto recorrente tem sido muito comum, por esse motivo este estudo uniu informações para assim contribuir com os profissionais de enfermagem, pois faz se necessário o conhecimento do tema, assim como suas causas e as estratégias para preveni-las e desta maneira poder prestar assistência da melhor forma, podendo evitar a perda gestacional.

Quanto aos limites deste estudo, está relacionada a falta de artigos que falem sobre o tema em si ou que tenham os mesmos objetivos que deste estudo. A maioria dos estudos encontrados, falam apenas de uma causa especifica sobre o abortamento de repetição, ou nem falam sobre elas, pois este é tema limitado e que precisa ser estudo ainda mais.

Como foi falado anteriormente, o levantamento de dados foi uma tarefa difícil pela limitação do tema, em função a essa restrição, recomenda que estudos futuros sejam realizados para obtenção de melhores resultados para maior conhecimento dos motivos que essas mulheres não conseguem ter uma gravidez eficaz, podendo assim os profissionais responsáveis intervirem de forma eficaz.

Portanto, concluímos que os resultados do presente estudo contribuíram para o entendimento e aprendizagem, pois o tema é essencial para compreensão dos profissionais de enfermagem. Levando em consideração esses aspectos, este estudo permite a melhor avaliação de riscos a mulher no seu período gravídico, assim como ao feto também. 


\section{Referências}

Bender Atik, R., Christiansen, O. B., Elson, J., Kolte, A. M., Lewis, S., ... \& Vermeulen, N. (2018). ESHRE guideline: recurrent pregnancy loss. Human Reproduction Open, (2).

Brasil, Ministério da Saúde. (2012). Atenção ao pré-natal de baixo risco. Cadernos de Atenção Básica, n. 32.

Brasil. (2012). Ministério da Saúde. Manual Técnico de Gestação de Alto Risco. Brasília.

Camayo, F. J. A., Martins, L. A. B., \& Cavalli, R. D. C. (2011). Perda gestacional retida: tratamento baseado em evidência. Femina, 49-56.

Febrasgo. Federação Brasileira das Associações de Ginecologia e Obstetrícia. (2010). Manual de Orientação Assistência ao Abortamento, Parto e Puerpério. São Paulo: Febrasgo.

Feliciano, N. B., Pradebon, V. M., \& de Lima, S. S. (2013). Enfermagem no pré-natal de baixo risco na estratégia Saúde da Família. Aquichan, 13(2), 261-269.

Gomes, C. B. D. A., Dias, R. D. S., Silva, W. G. B., Pacheco, M. A. B., Sousa, F. G. M. D., \& Loyola, C. M. D. (2019). Consulta de enfermagem no pré-natal: narrativas de gestantes e enfermeiras. Texto \& Contexto-Enfermagem, 28.

Jaslow, C. R., \& Kutteh, W. H. (2013). Effect of prior birth and miscarriage frequency on the prevalence of acquired and congenital uterine anomalies in women with recurrent miscarriage: a cross-sectional study. Fertility and sterility, 99(7), 1916-1922.

Junior, J., Giraldo, P. C., Gonçalves, A. K. S., \& Amaral, R. L. D. (2010). Qual deve ser o rastreamento de doenças infecciosas em casos de perda gestacional recorrente?: [revisão]. Femina.

Li, N., Wu, H. M., Hang, F., Zhang, Y. S., \& Li, M. J. (2017). Women with recurrent spontaneous abortion have decreased $25(\mathrm{OH})$ vitamin D and VDR at the fetal-maternal interface. Brazilian Journal of Medical and Biological Research, 50(11). 
Machin, R., Couto, M. T., Rocha, A. L. S., \& Costa, M. R. M. (2019). Formação médica e assistência aos processos de abortamento: a perspectiva de residentes de duas universidades públicas em São Paulo, Brasil. Interface-Comunicação, Saúde, Educação, 23.

Marqui, A. B. T. D. (2018). Anormalidades cromossômicas em abortos recorrentes por análise de cariótipo convencional. Revista Brasileira de Saúde Materno Infantil, 18(2), 265276.

Morales, C., Sánchez, A., Bruguera, J., Margarit, E., Borrell, A., Borobio, V., \& Soler, A. (2008). Cytogenetic study of spontaneous abortions using semi-direct analysis of chorionic villi samples detects the broadest spectrum of chromosome abnormalities. American Journal of Medical Genetics Part A, 146(1), 66-70.

Moura, S. G. D., Melo, M. M. M. D., César, E. S. R., Silva, V. C. L. D., Dias, M. D., \& Filha, M. D. O. F. (2015). Prenatal assistance carried out by nurse: a pregnant woman look. Revista de Pesquisa: Cuidado é Fundamental Online, 7(3), 2930-2938.

No, R. G. T. G. (2011). The investigation and treatment of couples with recurrent firsttrimester and second-trimester miscarriage. RCOG: London, UK.

Oliveira, M. A. D., Andari, V. C. M., Francisco, L. S., Ueno, J., Oliveira, R. M. D., \& Martinhago, C. D. (2011). Alterações no cariótipo que podem gerar infertilidade ou abortamento de repetição. Femina, 91-96.

OMS. (2011). Aborto inseguro: estimativas globais e regionais da incidência de aborto inseguro e mortalidade associada em 2008. 6ª ed. Genebra: Organização Mundial da Saúde.

OMS. (2013). Abortamento seguro: orientação técnica e de políticas para sistemas de saúde. Organização Mundial da Saúde (OMS).

Peixoto, S. (2014). Manual de assistência pré-natal. ed. São Paulo: Federação Brasileira das Associações de Ginecologia e Obstetrícia. 
Research, Society and Development, v. 9, n. 11, e53891110277, 2020

(CC BY 4.0) | ISSN 2525-3409 | DOI: http://dx.doi.org/10.33448/rsd-v9i11.10277

Practice Committee of the American Society for Reproductive Medicine. (2013). Definitions of infertility and recurrent pregnancy loss: a committee opinion. Fertility and sterility, 99(1), 63.

Ramos, K., Ferreira, A. L. C. G., \& de Souza, A. I. (2010). Women hospitalized due to abortion in a maternity teaching hospital in Recife, Brazil. Revista da Escola de Enfermagem da USP, 44(3), 602-607.

Rasmark R., E., Matthiesen, L., Rylance, R., \& Christiansen, O. B. (2017). Is the incidence of recurrent pregnancy loss increasing? A retrospective register-based study in Sweden. Acta Obstetricia et Gynecologica Scandinavica, 96(11), 1365-1372.

Rauber, F., Jacques, G., Feier, A. P., Piccinini, V. L., Michel, G. G., Petracco, R., ... \& Petracco, A. (2018). Abortamento de repetição: diagnóstico, investigação e manejo inicial. Acta méd.(Porto Alegre), 335-348.

Ribeiro, G. G., \& Alencar Júnior, C. A. Abortamento [Internet]. 2013 [citado 2013 Maio 1]. Sehnem, G., Saldanha de Saldanha, L., Arboit, J., Cammarano Ribeiro, A., \& Morais de Paula, F. (2020). Consulta de pré-natal na atenção primária à saúde: fragilidades e potencialidades da intervenção de enfermeiros brasileiros. Revista de Enfermagem Referência, (1).

Soares, M. C., Freitas, V. É., Cunha, A. R. R., Almeida, J. L. S., Souto, C. M. R. M., \& Dantas, R. A. (2012). Práticas de enfermagem na atenção às mulheres em situação de abortamento. Revista da Rede de Enfermagem do Nordeste, 13(1), 140-146.

Sociedade Beneficiente Israelita Brasileira (2019). Curetagem pós aborto. Guia do episódio de cuidado.

Souza, C. A. B., Schmitz, C., Genro, V. K., Martins, A., Scheffel, C., Oppermann, M. L., \& Cunha Filho, J. S. (2011). Histeroscopia ambulatorial em casos de abortamento consecutivo. Revista da Associação Médica Brasileira, 57(4), 404-408. 
Stirrat, G. M. (1990). Recurrent miscarriage I: definition and epidemiology. The Lancet, 336(8716), 673-675.

Ye, S. L., Gu, X. K., Tao, L. Y., Cong, J. M., \& Wang, Y. Q. (2017). Efficacy of different treatment regimens for antiphospholipid syndrome-related recurrent spontaneous abortion. Chinese Medical Journal, 130(12), 1395.

\section{Porcentagem de contribuição de cada autor no manuscrito}

$$
\text { Breno Pena Barros - 14,29\% }
$$

Carolynne Hernandez Barbosa - 14,29\%

Ellen Anny Pontes Moura dos Santos - 14,29\%

Esmeson Pereira da Costa - 14,29\%

Graciana de Sousa Lopes - 14,26\%

Roseane Gomes Ribeiro - 14,29\%

Welberson Soares de Souza - 14,29\% 